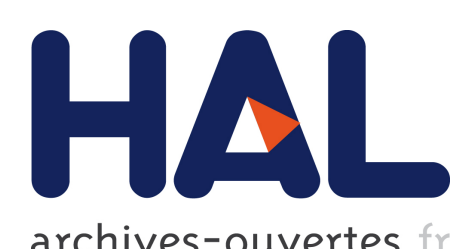

archives-ouvertes

\title{
VALORISATION DE DECHETS PLASTIQUES ET D'INDUSTRIE DU BOIS EN COMPOSITE MOULE A FROID : EFFET DES PARAMETRES DE FABRICATION SUR LES PROPRIETES MECANIQUES
}

Togbé Armel Amadji, Gerard Jean, Adjovi Codjo, Guibal Daniel, Doko

Kouandété Valéry

\section{To cite this version:}

Togbé Armel Amadji, Gerard Jean, Adjovi Codjo, Guibal Daniel, Doko Kouandété Valéry. VALORISATION DE DECHETS PLASTIQUES ET D'INDUSTRIE DU BOIS EN COMPOSITE MOULE A FROID : EFFET DES PARAMETRES DE FABRICATION SUR LES PROPRIETES MECANIQUES. Environnement, Ingénierie \& Développement, 2021, 1, pp.33-43. hal-03149395

\section{HAL Id: hal-03149395 \\ https://hal.archives-ouvertes.fr/hal-03149395}

Submitted on 23 Feb 2021

HAL is a multi-disciplinary open access archive for the deposit and dissemination of scientific research documents, whether they are published or not. The documents may come from teaching and research institutions in France or abroad, or from public or private research centers.
L'archive ouverte pluridisciplinaire HAL, est destinée au dépôt et à la diffusion de documents scientifiques de niveau recherche, publiés ou non, émanant des établissements d'enseignement et de recherche français ou étrangers, des laboratoires publics ou privés. 


\title{
VALORISATION DE DECHETS PLASTIQUES ET D'INDUSTRIE DU BOIS EN COMPOSITE MOULE A FROID : EFFET DES PARAMETRES DE FABRICATION SUR LES PROPRIETES MECANIQUES
}

\author{
AMADJI Togbé Armel ${ }^{1}$, GERARD Jean ${ }^{2}$, ADJOVI Edmond Codjo ${ }^{3}$, GUIBAL Daniel ${ }^{2}$, DOKO Kouandété Valéry ${ }^{1}$ \\ 1 Laboratoire d'Energétique et de Mécanique Appliquée, Université d'Abomey-Calavi, 01 BP 2009 Cotonou, Bénin \\ 2 UR BioWooEB, Cirad, TA B-73 114/16, 73 Rue J.F. Breton 34398, Montpellier, Cedex, France \\ 3 Ecole des Sciences et Techniques du Bâtiment et de la Route, Université Nationale des Sciences, Technologies, Ingénierie et \\ Mathématiques d'Abomey, B.P. 2282 Goho, Abomey, Bénin
}

*Auteur correspondant : AMADJI Togbé Armel, amadjitarmel@gmail.com

\section{RESUME}

Ce travail concerne le domaine du recyclage de déchets en matériaux. II rend compte d'une possibilité de valorisation de déchets plastiques et de produits connexes de l'industrie du bois dans la construction. L'étude vise à donner une valeur ajoutée à un composite bois-plastique élaboré suivant un procédé de moulage par compression à froid, par l'amélioration de ses propriétés mécaniques. A travers un essai de flexion, l'influence de deux paramètres de fabrication - pression de compactage et température de séchage postmoulage - sur les propriétés mécaniques, a été évaluée dans le cas d'un composite à base de sciures de bois tropicaux venant du Bénin et de polystyrène (CBPo) recyclés. Il en ressort que la pression de compactage et la température de séchage, sont deux facteurs essentiels à considérer lors de l'évaluation des performances mécaniques du composite. L'augmentation de la pression de compactage jusqu'à une valeur limite améliore significativement les propriétés du matériau. Les propriétés optimales du CBPo ont été obtenues à la pression de $7 \mathrm{MPa}$. Le séchage post-moulage à $100^{\circ} \mathrm{C}$, a également amélioré de façon significative les performances du composite. Aussi, par ses nouvelles performances mécaniques, le CBPo se repositionne par rapport à d'autres matériaux existants permettant ainsi d'en envisager de nouvelles applications dans la construction.

MOTS-CLES : recyclage des déchets, composite boispolystyrène, effet des paramètres de fabrication, pression de compactage, température de séchage, propriétés mécaniques.

\section{ABSTRACT}

This work concerns the field of recycling of waste into building materials. It reports on the possibility of recovering plastic waste and related products from the wood industry, in construction. The objective of the study is to give added value to wood-plastic composites produced using the cold compression molding process, by improving their mechanical properties. Through the bending test, the influence of two manufacturing parameters - compaction pressure and drying temperature after molding - on the mechanical properties, was evaluated for the case of a composite based on tropical wood sawdust coming from Benin and recycled polystyrene (CBPo). It appears that the compaction pressure and the drying temperature are essential factors to consider when evaluating the mechanical performance of the composite. Increasing the compaction pressure to a limit value significantly improves the properties of the material. The optimal properties of CBPo were obtained at a pressure of $7 \mathrm{MPa}$. Post-forming drying at $100^{\circ} \mathrm{C}$ also significantly improved the performance of the composite. In addition, thanks to its enhanced new mechanical properties, the CBPo is repositioned relatively to other existing materials, thus making it possible to think about new applications in construction.

KEYWORDS: waste recycling, wood-polystyrene composite, effect of manufacturing parameters, compaction pressure, drying temperature, mechanical properties. 


\section{INTRODUCTION}

La transformation de déchets en matériaux est une forme de valorisation qui présente un double avantage en contribuant à l'assainissement de l'environnement, mais également à la mise à disposition de nouveaux matériaux dans le domaine de la construction. Cependant, dans le but de satisfaire des besoins en constructions sécurisées et économiques, la valorisation des déchets doit viser des matériaux de plus en plus performants. Dans cet objectif, différents types de matériaux composites sont développés, notamment les multicouches, les composites à renfort fibreux ou particulaires, dont les propriétés résultent d'associations de propriétés de leurs constituants. Parmi les composites, les bois plastiques (CBP) sont de plus en plus utilisés du fait non seulement de leur durabilité élevée, leur faible entretien et leur résistance et rigidité acceptables (Patel et Rawat, 2017) mais aussi pour la gestion appropriée de leur fin de vie (Le Duigou et al., 2016). Toutefois, les CBP sont confrontés à des problèmes de baisse de performances en raison de la nature des phases (constituants) souvent à l'origine d'une insuffisance d'adhésion à leur interface (Poletto et al., 2011). La résolution de ces problèmes nécessite en général une maîtrise parfaite de chaque étape du processus de fabrication.

La fabrication des CBP utilise plusieurs procédés dont l'extrusion (le plus courant), le moulage par injection, le thermoformage (Matuana et Stark, 2015 ; Almusawi, 2017). Un autre procédé présenté récemment par plusieurs auteurs (Agoua et al., 2013 ; Masri et al., 2018) est le moulage par compression à froid. Ce procédé est utilisé pour recycler des déchets de polystyrène expansé d'emballage (PSE) et des sciures de bois. II consiste d'abord à préparer le composite par mélange de sciures à une matrice adhésive formée par dissolution de PSE dans un solvant organique approprié, une méthode de recyclage du PSE par voie chimique (Gil-Jasso et al., 2019). Ensuite, le composite est mis en forme par simple compactage à la presse mécanique, puis exposé à l'air libre pour séchage. Le recyclage de déchets de PSE devenant une préoccupation majeure au niveau mondial (Mangalara et Varughese, 2016), leur transformation en matériau selon le procédé de moulage par compression, présente plusieurs avantages pour les pays en développement, comme le Bénin. D'une part, l'utilisation de déchets de PSE pour la production de matériaux composites, est d'une grande importance économique et environnementale (Serranti et al., 2011), d'autre part, le procédé de transformation est accessible, adapté aux besoins et aux possibilités des populations locales (Agoua et al., 2013).
La technologie de production des CBP fait appel à des concepts relatifs à la compatibilité des polymères, aux procédés de fabrication, avec d'importants défis d'amélioration, voire d'optimisation des propriétés du composite final. Ces propriétés dépendent principalement des paramètres choisis pour la formulation et la fabrication du matériau. Les paramètres relatifs à la formulation du matériau (nature et proportion des phases, morphologie du renfort/charge) sont souvent pris en compte dans les travaux d'étude des nouveaux composites. Elles y sont présentées comme principaux facteurs permettant de juger du potentiel de ces matériaux. Depuis l'avènement des composites, beaucoup d'attention a été accordée aux paramètres relatifs à la formulation, quel que soit le type de procédé de fabrication utilisé (Kokta et al., 1983 ; Stark et Rowlands, 2003 ; Bessa et al., 2017). Cependant, le constat n'est pas exactement le même pour ce qui concerne l'influence des paramètres de fabrication sur les performances des composites.

Certes, depuis plusieurs décennies, la question de l'influence des paramètres de fabrication sur les propriétés des composites extrudés, a fait l'objet de beaucoup de travaux (Crevecoeur et Groeninckx, 1993 ; Sallih et al., 2014). Le Duigou et al. (2016) rapportent que, de plus en plus, des études sont menées sur les composites extrudés pour promouvoir le développement de ce type de méthodes. En ce qui concerne les composites moulés par injection, beaucoup de travaux ont été également conduits sur l'étude de l'influence des paramètres de fabrication (O'Dowd et al., 2006 ; Wong et al., 2008). Dans la catégorie des composites thermoformés, les travaux d'Almusawi (2017) ont permis de mettre en relief l'influence des paramètres de fabrication sur les propriétés de ces matériaux.

Cependant, contrairement aux différentes méthodes citées ci-dessus et aux connaissances mises en avant par ces auteurs, l'étude de l'influence des paramètres de fabrication sur les propriétés mécaniques des composites moulés par compression à froid, en particulier ceux à base de farine de bois et de colle de PSE recyclé (CBPo), n'a encore fait l'objet d'aucune recherche. Pourtant, la nécessité $d^{\prime}$ 'une telle étude a été exprimée par Masri et al. (2018), à la suite de leurs travaux sur un composite à base de PSE et de fibres de branches de palmier dattier, moulé par compression à froid. Pour ce procédé de mise en forme, on cherche à réduire la porosité dans le matériau, de façon à le faire passer de l'état foisonné, c'est-à-dire non compact, à forte porosité, avec faibles propriétés mécaniques, à un état plus compacté, minimisant les porosités. Ceci est indispensable parce que la porosité à l'intérieur du matériau influe sur ses propriétés (Madsen, 2004). 
L'objectif du présent travail est d'étudier l'influence des paramètres de fabrication sur les propriétés mécaniques de CBPo (à base de déchets de PSE, utilisés pour une colle, et de farines de bois recyclées) moulés par compression à froid. Les paramètres de fabrication pris en compte dans l'étude sont la pression de compactage et la température de séchage post-formage de CBPo.

\section{MATERIELS ET METHODES}

\subsection{Elaboration des composites}

Les CBPo ont été fabriqués à partir de polystyrène expansé issu d'emballages (PSE) recyclés, de solvant et de sciure de bois. Le PSE utilisé a été collecté dans des magasins de vente de motocycles, d'appareils électroménagers et sur des décharges de déchets de la ville de Cotonou (Bénin). Il a été nettoyé puis réduit en fragments de taille $\leq 5 \mathrm{~cm}$. La dissolution des morceaux de PSE dans du solvant (essence) donne une colle (Agoua et al., 2013). L'essence utilisée est en moyenne composée de 20 à $30 \%$ d'alcanes, $5 \%$ de cyclo alcanes, 30 à $45 \%$ d'alcènes, 30 à $45 \%$ d'hydrocarbures de la famille du benzène. Le rapport de la masse de PSE à celle du solvant, adopté pour raison d'efficience, est de 1,22. La sciure utilisée est celle du bois de Gmelina (Gmelina arborea) obtenue auprès de l'Office national du bois (ONAB). Sa distribution granulométrique présentée à la Figure 1 montre qu'elle est constituée principalement de particules de taille comprise entre $0,315 \mathrm{~mm}$ et $0,630 \mathrm{~mm}$ (refus sur tamis de 0,315). Cette classe granulaire a été utilisée dans la présente étude. Les sciures du bois de Teck (Tectona grandis) et d'Afzelia (Afzelia africana), de même granulométrie, ont aussi été utilisées, mais dans une moindre mesure. Leurs distributions granulométriques sont également présentées à la Figure 1. Avant leur utilisation, les sciures ont été séchées à l'étuve à $103 \pm 2^{\circ} \mathrm{C}$ jusqu'à masse constante puis emballées dans des films en polyéthylène basse densité, afin de les préserver de I'humidité de l'air préjudiciable aux performances mécaniques du matériau final (Matuana and Stark, 2015). Les composites ont été élaborés avec une proportion en sciures de $40 \%$. Cette proportion a été retenue parce qu'elle permet d'avoir un bon compromis entre homogénéité et résistance mécanique du matériau. L'homogénéité des CBPo se dégrade par formation d'agglomérats lorsque la proportion de sciure diminue dans celui-ci et la résistance mécanique baisse quand la teneur en sciure augmente. La colle a été mélangée avec les sciures à l'aide d'un malaxeur de marque Hobart (modèle $A 200$, pale de type " $B$ "; fréquence de rotation de la pale, $107 \mathrm{trs}^{\mathrm{min}}{ }^{-1}$; fréquence de rotation de l'axe de la pale, $\left.61 \mathrm{trs} \cdot \mathrm{min}^{-1}\right)$. Les composites ainsi obtenus ont été étalés et exposés à l'air libre pendant vingt minutes, avant d'être moulés à froid par compression à l'aide d'un moule de dimensions intérieures $138 \times 571 \mathrm{~mm}^{2}$ et d'une presse mécanique de capacité 150 tonnes. Les plaques ont été moulées à une épaisseur finale de $10 \mathrm{~mm}$ (valeur nominale). Elles ont été ensuite séchées à l'air libre $\left(25^{\circ} \mathrm{C}\right.$ environ) pendant trois semaines, puis certaines ont été conditionnées à l'étuve (à $60^{\circ} \mathrm{C}$ et $100^{\circ} \mathrm{C}$ ) pendant 24 heures puis à nouveau exposées à l'air libre pendant $72 \mathrm{~h}$ avant d'être testées.

\subsection{Echantillonnage}

Pour analyser l'influence de paramètres de fabrication sur les propriétés mécaniques des CBPo, deux facteurs d'influence que sont la pression de compactage et la température, ont été étudiés. Dans un premier temps, l'étude de l'influence de la pression de compactage sur les performances mécaniques des $\mathrm{CBP}$ a été réalisée. Cinq échantillons (plaques) à base de sciure de Gmelina ont été confectionnés pour les niveaux de pression 1, 3, 5, 7 et $8 \mathrm{MPa}(1 \mathrm{MPa}=10 \mathrm{bars})$. Ces valeurs de pression ont été retenues à la suite d'un essai de pressage qui a montré le comportement du CBPo (dans la direction de la charge) lors du moulage. L'évolution du taux de compactage en fonction de la pression de compactage a alors été analysée. Le taux de compactage, $T c$, est donné par la relation (1) :

$T_{c}(\%)=\frac{e_{i}-e_{t}}{e_{i}} \times 100$

$e_{t}$ : épaisseur de l'échantillon à un instant t pour une valeur donnée de charge,

$e_{i}$ : épaisseur initiale de l'échantillon.

Tableau 1. Températures testées pour les propriétés mécaniques des 3 types de bois (pression de compactage $5 \mathrm{MPa})$.

\begin{tabular}{|c|c|c|}
\hline $\begin{array}{c}\text { Espèce de } \\
\text { bois }\end{array}$ & $\begin{array}{c}\text { Température } \\
\left({ }^{\circ} \mathrm{C}\right)\end{array}$ & $\begin{array}{c}\text { Références des } \\
\text { échantillons }\end{array}$ \\
\hline \multirow{3}{*}{ Gmelina } & 25 & G_5MPa_25 ${ }^{\circ} \mathrm{C}$ \\
\hline & 60 & $\mathrm{G} \_5 \mathrm{MPa}=60^{\circ} \mathrm{C}$ \\
\hline & 100 & $\mathrm{G}$ 5 $5 \mathrm{MPa}$ _ $100^{\circ} \mathrm{C}$ \\
\hline \multirow{3}{*}{ Teck } & 25 & $\mathrm{~T} \_5 \mathrm{MPa} \_25^{\circ} \mathrm{C}$ \\
\hline & 60 & $\mathrm{~T}_{-} 5 \mathrm{MPa} \mathrm{MP}^{\circ} \mathrm{C}$ \\
\hline & 100 & T_5MPa_100 ${ }^{\circ} \mathrm{C}$ \\
\hline \multirow{3}{*}{ Afzelia } & 25 & A_5MPa_25 ${ }^{\circ} \mathrm{C}$ \\
\hline & 60 & A_5MPa_60 $60^{\circ} \mathrm{C}$ \\
\hline & 100 & A_5MPa_ $100^{\circ} \mathrm{C}$ \\
\hline
\end{tabular}


Dans un second temps, l'effet de la température a été étudié en couplage avec l'espèce de bois utilisée (provenance de la sciure), pour le niveau de pression de $5 \mathrm{MPa}$; cette valeur limitée de pression, a été choisie afin de limiter les déformations préjudiciables au moule utilisé. Le plan expérimental utilisé est indiqué dans le tableau 1. Les éprouvettes ont été découpées à la scie circulaire. Cinq éprouvettes de flexion par échantillon (pour un total de 13 échantillons), de dimensions $291 \times 76 \times 10 \mathrm{~mm}^{3}$ ont été utilisées, conformément à la norme ASTM D 103712 (2012). La mesure de densité et de porosité a été effectuée sur les échantillons pour lesquels a été étudiée l'influence de la pression de compactage ; six éprouvettes $\left(30 \times 10 \times 10 \mathrm{~mm}^{3}\right)$ ont été utilisées par échantillon.
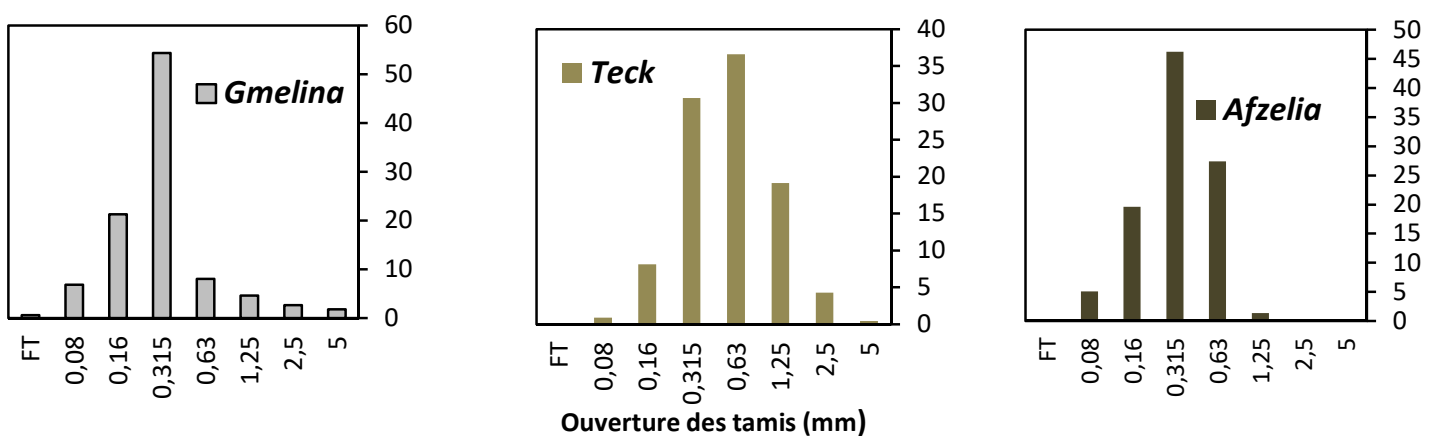

Figure 1. Distribution granulométrique des sciures de Gmelina, Teck et Afzelia.

\subsection{Essai de flexion}

Les propriétés mécaniques ont été déterminées à I'aide d'un essai de flexion trois points, selon la norme ASTM D 1037-12 (2012). Une machine d'essai de marque MTS instrumentée avec un capteur de $250 \mathrm{kN}$, piloté par ordinateur avec enregistrement des valeurs de charge et flèche, a été utilisée. La vitesse de déplacement de la traverse pendant l'essai était de 4 $\mathrm{mm} \cdot \mathrm{min}^{-1}$. La Figure 2 montre le montage utilisé. La contrainte maximale (ou module de rupture MOR) et le module d'élasticité (MOE) ont été déterminés à I'aide des équations (2) et (3) :

$$
\begin{aligned}
& M O R=\frac{3 P_{\max } L}{2 b h^{2}} \\
& M O E=\frac{L^{3}}{4 b h^{3}} \frac{\Delta P}{\Delta y}
\end{aligned}
$$

avec :

Pmax : charge maximale, en $\mathrm{N}$

$L$ : distance entre appuis, en $\mathrm{mm}$

b : largeur de l'éprouvette, en $\mathrm{mm}$

$\mathrm{h}$ : épaisseur de l'éprouvette, en $\mathrm{mm}$

$\frac{\Delta P}{\Delta y}$ : pente du domaine linéaire de la courbe chargeflèche à mi-portée, déterminée par régression linéaire pour les valeurs de charges comprises entre $10 \%$ et $40 \%$ de la charge maximale.

\subsection{Mesures des masses volumiques et de la porosité}

La masse volumique particulaire $\rho_{p}$ est le rapport entre la masse d'un échantillon et le volume de la matière qui le constitue. Le volume de la matière constitutive est le volume total de l'échantillon diminué du volume occupé par les pores. Le volume de la matière constitutive a été déterminé à l'aide d'un pycnomètre à déplacement de gaz (hélium), modèle AccuPyc II 1340, avec une cellule pour éprouvette de $10 \mathrm{~cm}^{3}$ (Figure 3). Ces éprouvettes ont été préalablement déshumidifiées dans un dessiccateur à silicagel. Pour chaque éprouvette, 20 cycles de mesure du volume ont été réalisés.

La masse volumique apparente $\rho_{b}$ est le rapport de la masse de l'échantillon par son volume d'enveloppe. Le volume d'enveloppe (ou volume total) a été déterminé par la méthode de la double pesée (utilisation du principe de la poussée d'Archimède). Les éprouvettes ont été pesées à l'aide d'une balance digitale, modèle Mettler AE 160, de précision $0,0001 \mathrm{~g}$.

La porosité d'un matériau exprime la proportion du volume des pores dans celui-ci par rapport à son volume total. Elle est égale au rapport entre la différence des masses volumiques particulaire et apparente, et la masse volumique particulaire du matériau. Pour chaque éprouvette, la porosité $p$ (en pourcentage) a été calculée suivant l'équation (4) :

$p(\%)=100\left(\frac{\rho_{p}-\rho_{b}}{\rho_{p}}\right)$

Pour chaque échantillon, la moyenne arithmétique et l'écart-type des masses volumiques et des porosités, ont été déterminés. 


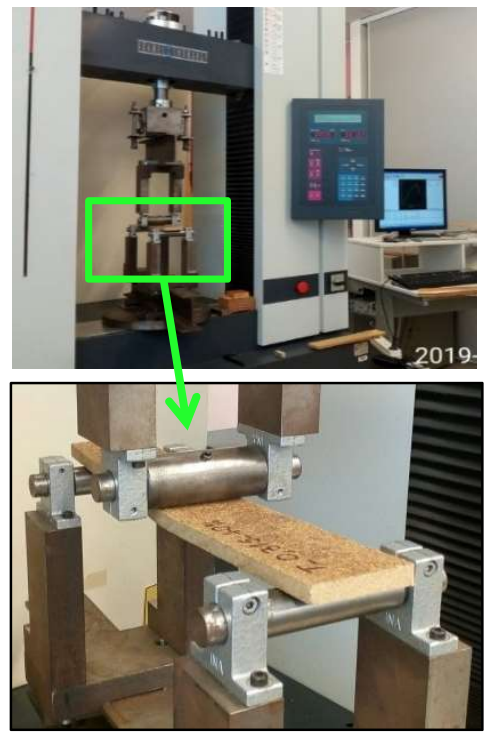

Figure 2. Dispositif expérimental pour l'essai de flexion

\section{RESULTATS ET DISCUSSION}

\subsection{Comportement des composites au moulage}

L'étude de l'influence de la contrainte de compactage sur les propriétés mécaniques des composites nécessite dans un premier temps la connaissance du comportement du composite lors du moulage. Les composites à l'état foisonné (état après exposition à I'air) sont compactés à froid à la presse mécanique. La Figure 3 montre l'évolution du taux de compactage en fonction de la contrainte de compactage.

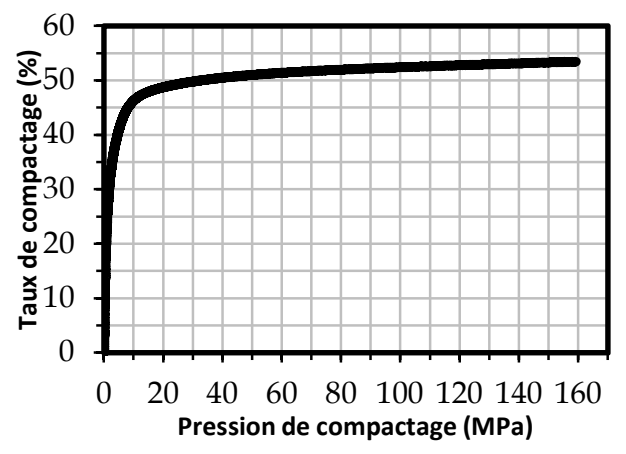

Figure 3. Evolution du taux de compactage en fonction de la pression.

On observe une première phase de compactage d'environ $25 \%$ sous une contrainte négligeable, puis une deuxième phase caractérisée par un compactage variant de 25 à $45 \%$ sous une contrainte de 1 à $10 \mathrm{MPa}$ et enfin, une dernière phase de stabilisation du compactage pour des valeurs de contrainte supérieures à $10 \mathrm{MPa}$. Ce comportement est analogue à celui du composite à base de chevronnette et de pâte de polystyrène développé par Almusawi (2017).
3.2. Effet de la pression de compactage sur les propriétés en flexion

L'essai de flexion trois points permet de connaître le comportement mécanique du matériau et d'obtenir la contrainte maximale et le module d'élasticité, en flexion. La Figure 4 montre l'influence de la pression de compactage sur le comportement mécanique des CBPo. On note que les CBPo ont un comportement quasi-fragile pour toutes les valeurs de pression testées. La fragilité des composites bois-plastique avec des particules a déjà été observée dans la littérature (Binhussain et El-Tonsy, 2013 ; Masri et al., 2018).

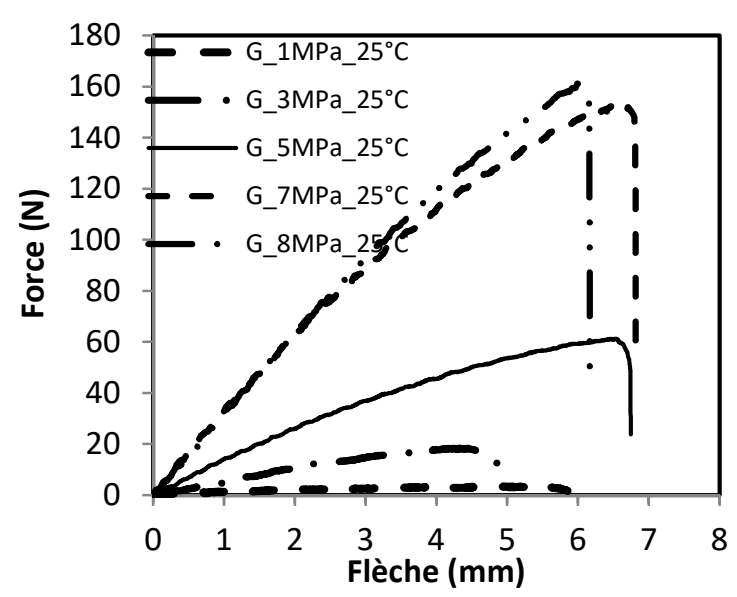

Figure 4. Courbe force-flèche du CBPo en flexion, pour différentes valeurs de pression de compactage, (échantillons à base de sciure de Gmelina séchés à $25^{\circ} \mathrm{C}$ )

La Figure 5 montre respectivement l'évolution du module d'élasticité (MOE) et celle du module de rupture (MOR) (ou contrainte maximum) des CBPo, en fonction de la pression de compactage. On observe que le MOE croît avec l'augmentation de la pression de compactage jusqu'à $7 \mathrm{MPa}$ puis se stabilise après cette valeur de pression. La même observation est faite pour le MOR qui croît, puis se stabilise à partir de la pression de $7 \mathrm{MPa}$. Ainsi, dans l'ensemble, les propriétés mécaniques des CBPo s'améliorent avec l'augmentation de la pression de compactage jusqu'à $7 \mathrm{MPa}$, valeur de pression à partir de laquelle aucune amélioration sensible n'est observée. D'après le tableau 2, on peut remarquer que le MOE croît de 104,94 MPa (pour une pression de $1 \mathrm{MPa}$ ) à 1352,2 $\mathrm{MPa}$ (pour une pression de $7 \mathrm{MPa}$ ), soit une valeur de MOE multipliée par 13 . Une forte augmentation est aussi notée au niveau du MOR qui passe de 0,25 MPa (pour une pression de $1 \mathrm{MPa}$ ) à 7,82 $\mathrm{MPa}$ (pour une pression de $7 \mathrm{MPa}$ ), soit une valeur multipliée par près de 30 . 
Tableau 2. Influence de la pression de compactage sur les propriétés des CBPo (échantillons à base de sciure de Gmelina et séchés à $25^{\circ} \mathrm{C}$ ).

\begin{tabular}{ccccc}
\hline $\begin{array}{c}\text { Pression de } \\
\text { compactage (MPa) }\end{array}$ & $\begin{array}{c}\text { Masse volumique } \\
\text { apparente }\left(\mathbf{k g} / \mathbf{m}^{3}\right)\end{array}$ & $\begin{array}{c}\text { Porosité } \\
(\%)\end{array}$ & $\begin{array}{c}\text { MOR } \\
(\mathbf{M P a})\end{array}$ & $\begin{array}{c}\text { MOE } \\
(\mathrm{GPa})\end{array}$ \\
\hline $\mathbf{1}$ & $523,56 \pm 10,33$ & $57,14 \pm 0,91$ & $0,25 \pm 0,06$ & $0,105 \pm 0,005$ \\
$\mathbf{3}$ & $571,76 \pm 23,25$ & $53,07 \pm 1,75$ & $1,04 \pm 0,21$ & $0,202 \pm 0,021$ \\
$\mathbf{5}$ & $636,62 \pm 16,40$ & $47,66 \pm 1,67$ & $3,88 \pm 0,74$ & $0,634 \pm 0,064$ \\
$\mathbf{7}$ & $758,26 \pm 16,84$ & $37,49 \pm 1,45$ & $7,82 \pm 0,99$ & $1,352 \pm 0,067$ \\
$\mathbf{8}$ & $758,68 \pm 17,48$ & $37,43 \pm 1,33$ & $7,84 \pm 1,30$ & $1,309 \pm 0,136$ \\
\hline
\end{tabular}

La valeur optimale de la pression de compactage qui donne les meilleures performances mécaniques du CBPo est donc de $7 \mathrm{MPa}$. Les performances mécaniques du CBPo à $7 \mathrm{MPa}$, sont supérieures (au moins deux fois plus élevées) à celles d'un composite avec matrice PSE et fibres de coco, obtenu avec une pression de $1 \mathrm{MPa}$ tel qu'indiqué par Masri et al. (2018); alors que les fibres, comparées aux particules, sont bien connues pour augmenter les propriétés des composites bois-plastiques (Ansari et al., 2017 ; Borsoi et al., 2014). Ceci démontre le pouvoir améliorant de la pression de compactage sur les propriétés mécaniques des composites moulés sous pression.

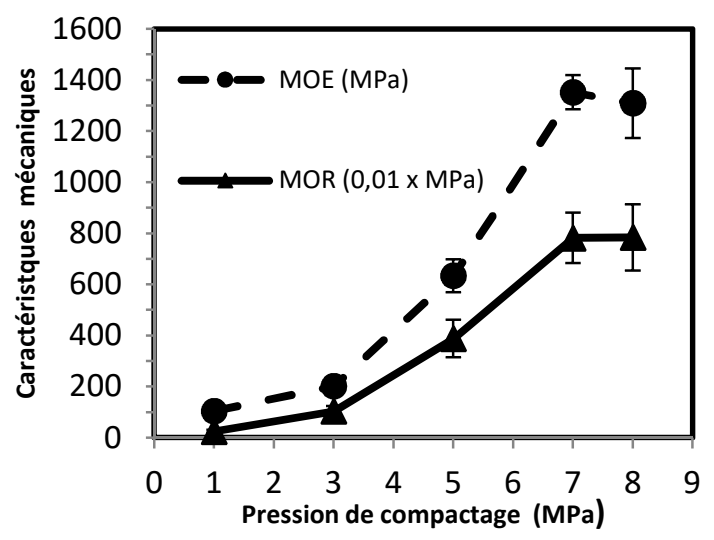

Figure 5. Evolution des caractéristiques mécaniques du CBPo en fonction de la pression de compactage, (échantillons à base de sciure de Gmelina séchés à $25^{\circ} \mathrm{C}$ ).

La Figure 6 montre l'évolution de la masse volumique des CBPo en fonction de la pression de compactage. On observe, comme le présage le résultat du test de pressage (Figure 4), une densification des CBPo qui se stabilise à partir de la pression de $7 \mathrm{MPa}$ pour une masse volumique limite de $758 \mathrm{~kg} \cdot \mathrm{m}^{-3}$ environ (tableau 2). De plus, on note sur le tableau 2 une amélioration des propriétés mécaniques lorsque la masse volumique augmente avec une forte corrélation entre les deux propriétés. Cette tendance est en accord avec celle décrite dans la littérature (Almusawi, 2017).

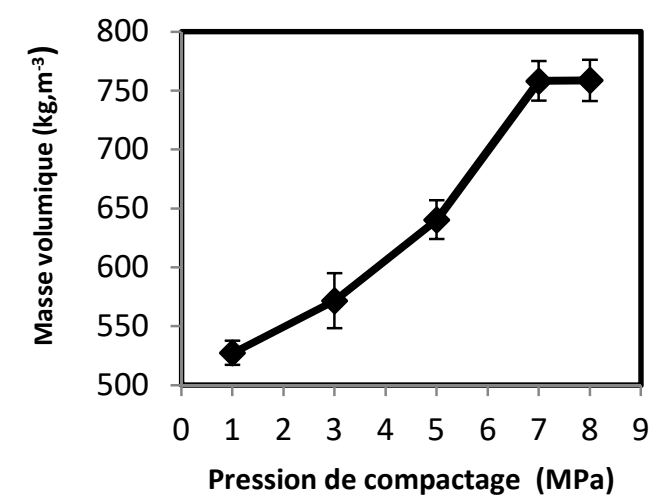

Figure 6. Evolution des caractéristiques mécaniques du CBPo en fonction de la pression de compactage, (échantillons à base de sciure de Gmelina séchés à $25^{\circ} \mathrm{C}$ ).

Pendant le moulage par compression à froid d'un matériau, l'objectif est souvent de réduire la porosité à une valeur minimale. Les résultats de la mesure de porosité du CBPo, sont présentés sur la Fig. 7 .

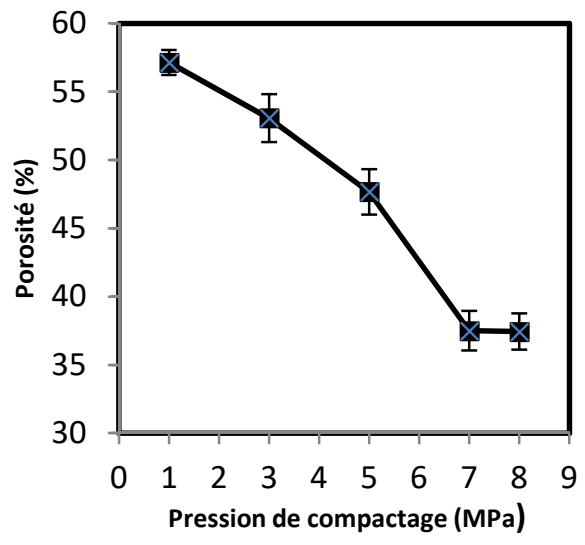

Figure 7. Evolution de la porosité des CBPo en fonction de la pression de compactage, (échantillons à base de sciure de Gmelina séchés à $25^{\circ} \mathrm{C}$ ).

On observe que la porosité décroît avec l'augmentation de la pression de compactage et se stabilise à partir de $7 \mathrm{MPa}$, ce qui va dans le sens de l'évolution de la masse volumique et des propriétés mécaniques. De plus, la Figure 8 montre une très forte dépendance entre les propriétés mécaniques et la 
porosité des CBPo, pour les valeurs de pression inférieures à $7 \mathrm{MPa}$. Ceci démontre non seulement l'effet négatif de la porosité sur les propriétés mécaniques des CBPo, comme l'ont signalé plusieurs auteurs (Madsen, 2004 ; Animpong et al., 2017), mais surtout la nécessité de reconsidérer la pression de compactage comme facteur d'influence significatif qui améliore les propriétés mécaniques des CBPo.

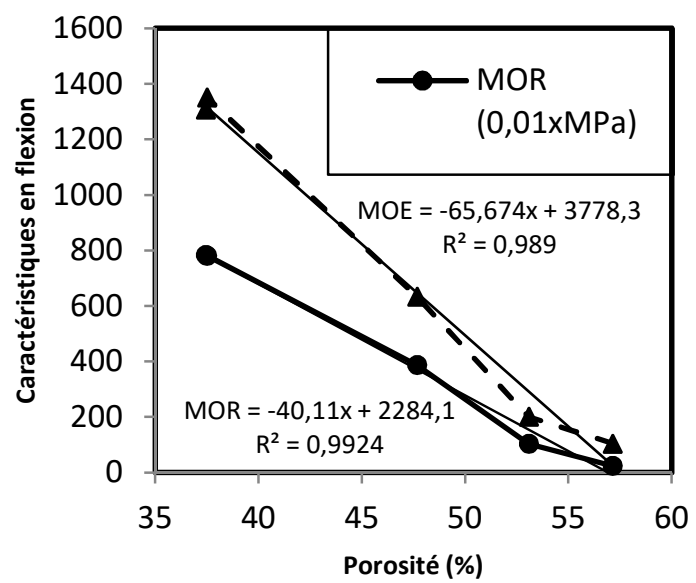

Figure 8. Evolution du MOE et du MOR des CBPo en fonction de la pression de compactage, (échantillons à base de sciure de Gmelina séchés à $25^{\circ} \mathrm{C}$ ).

\subsection{Effet de la température sur les propriétés en flexion}

L'essai de flexion est aussi utilisé pour tester l'effet de la température de séchage et de l'espèce de bois sur les propriétés mécaniques des CBPo. La Figure 9 montre l'influence de la température sur le comportement mécanique des CBPo.

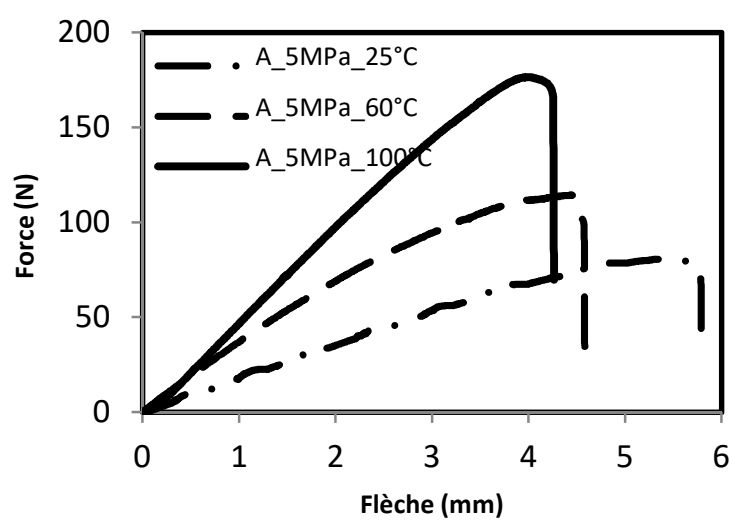

Figure 9. Courbe type force-flèche de CBPo en flexion à différentes température (échantillons compactés à $5 \mathrm{MPa})$

On note que les propriétés mécaniques changent lorsque la température change. On observe également une variation des propriétés du composite selon l'espèce de bois utilisée (Figure 10). En revanche, le mécanisme de rupture ne change pas et le comportement des CBPo reste globalement quasifragile pour les valeurs de température étudiées et pour toutes les espèces de bois testées.

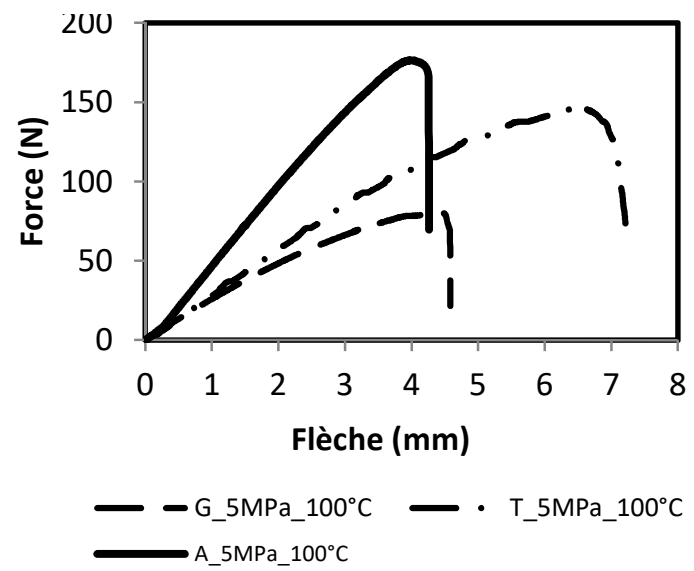

Figure 10. Courbe type force-flèche de CBPo en flexion pour différentes espèces séchées à $100^{\circ} \mathrm{C}(\mathrm{G}: \mathrm{Gmelina}$; $\mathrm{A}$ : Afzelia ; $\mathrm{T}$ :Teck ; (échantillons compactés à $5 \mathrm{MPa})$.

La Figure 11 permet de comparer les valeurs des caractéristiques mécaniques pour les niveaux de température étudiés et selon l'espèce de bois. Pour toutes les espèces étudiées, on observe que l'augmentation de la température améliore aussi bien le $\mathrm{MOE}$ que le $\mathrm{MOR}$ des $\mathrm{CBPo}$. De $25^{\circ} \mathrm{C}$ à $100^{\circ} \mathrm{C}$, la valeur du MOE du CBPo à base de sciure de Gmelina a doublé, passant de $0,60 \mathrm{GPa}$ à $1,21 \mathrm{GPa}$, celui du CBPo à base de Teck a augmenté de plus de 1,5 fois en passant de 0,80 GPa à 1,75 GPa (tableau 3). Quant au CBPo à base de sciure $d^{\prime} A f z e l i a$, la valeur du MOE a triplé, passant de 0,80 GPa à 2,26 GPa.

Dans l'ensemble, la valeur de MOE la plus élevée $(2,26 \mathrm{GPa})$ est enregistrée pour le composite à base de sciure d'Afzelia, étuvé à $100^{\circ} \mathrm{C}$. En ce qui concerne le $\mathrm{MOR}$, il a globalement doublé pour chacune des trois catégories de $\mathrm{CBPo}$. De $25^{\circ} \mathrm{C}$ à $100^{\circ} \mathrm{C}$, le $\mathrm{MOR}$ passe de 2,71 $\mathrm{MPa}, 3,87 \mathrm{MPa}$ et 4,61 $\mathrm{MPa}$ à 6,76 $\mathrm{MPa}$, 6,77 $\mathrm{MPa}$ et 8,93 $\mathrm{MPa}$ respectivement pour le $\mathrm{CBPo}$ à base de sciure de Gmelina, de Teck et d'Afzelia (tableau 3). La valeur de MOR la plus élevée $(8,93$ $\mathrm{MPa}$ ) correspond au composite à base de sciure d'Afzelia étuvé à $100^{\circ} \mathrm{C}$. On explique l'effet positif de la température de séchage sur les propriétés des CBPo par une meilleure élimination du solvant.

La réduction par évaporation du solvant favorise la solidification de la matrice PSE et l'amélioration de l'adhésion à l'interface bois-PS. L'amélioration de l'adhésion à l'interface bois-PS se manifeste par une contribution de la phase bois à l'amélioration des propriétés mécaniques du composite. 


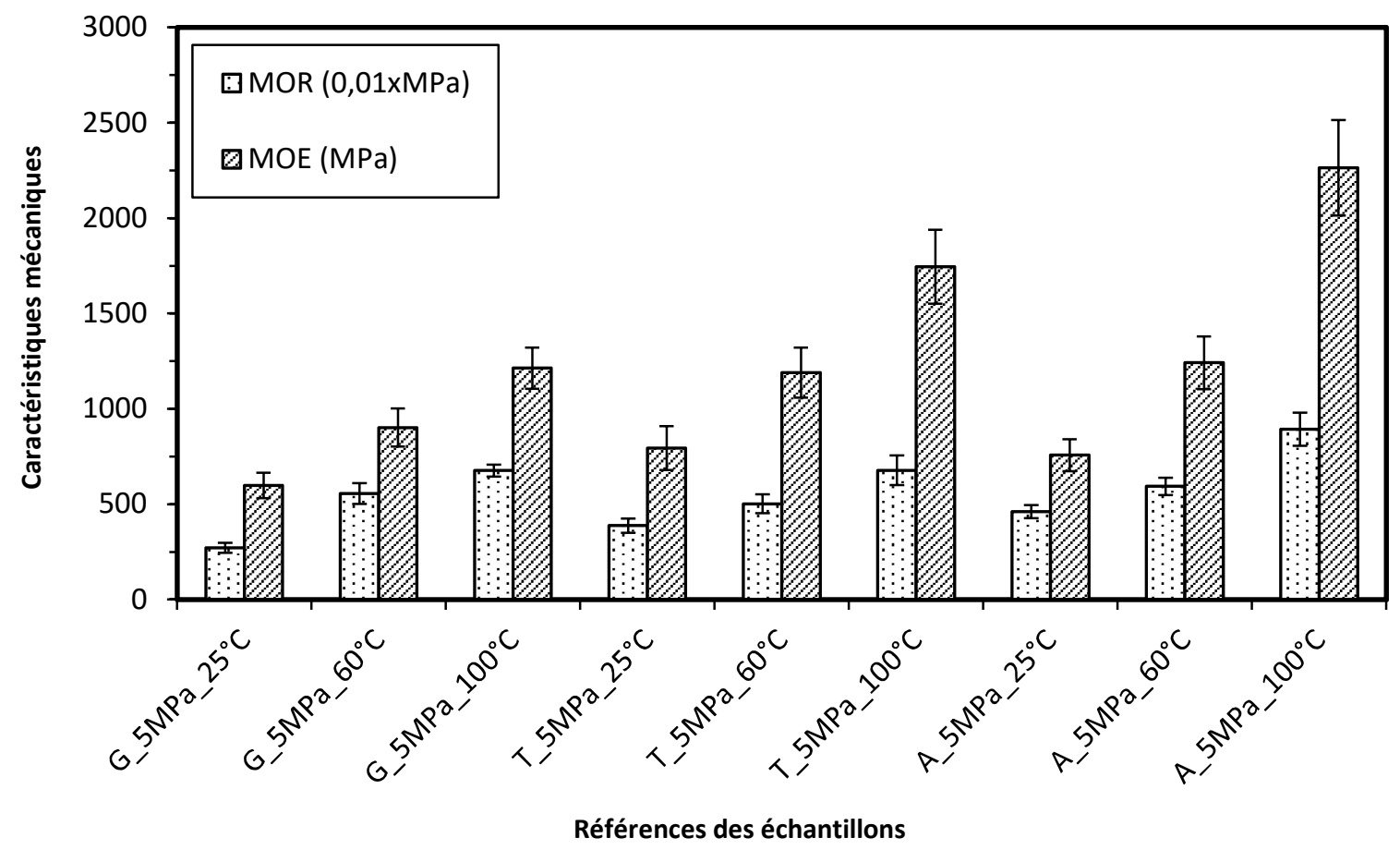

Figure 11. Valeurs comparées de MOE et MOR des CBPo, pour les 3 températures et les 3 espèces de bois (échantillons compactés à $5 \mathrm{MPa}$ ).

Tableau 3. Propriétés comparées des CBPo et de matériaux similaires

\begin{tabular}{|c|c|c|c|}
\hline Echantillons & $\rho\left(\right.$ kg. m $\left.{ }^{-3}\right)$ & MOE (GPa) & MOR (MPa) \\
\hline G_7 $\mathrm{MPa}$-25 $5^{\circ} \mathrm{C}$ & $758,68 \pm 17.48$ & $1,35 \pm 0,07$ & $7,82 \pm 0,99$ \\
\hline $\mathrm{G}_{-} 5 \mathrm{MPa} \_25^{\circ} \mathrm{C}$ & / & $0,60 \pm 0,06$ & $2,71 \pm 0,26$ \\
\hline T_5MPa_25 ${ }^{\circ} \mathrm{C}$ & / & $0,79 \pm 0,12$ & $3,87 \pm 0,38$ \\
\hline A_5 $\mathrm{MPa} \_25^{\circ} \mathrm{C}$ & / & $0,79 \pm 0,08$ & $4,61 \pm 0,35$ \\
\hline $\mathrm{G} \_5 \mathrm{MPa} \_100^{\circ} \mathrm{C}$ & / & $1,21 \pm 0,11$ & $6,76 \pm 0,31$ \\
\hline T_5MPa_ $100^{\circ} \mathrm{C}$ & / & $1,75 \pm 0,19$ & $6,77 \pm 0,78$ \\
\hline A_5MPa_ $100^{\circ} \mathrm{C}$ & / & $2,26 \pm 0,25$ & $8,93 \pm 0,87$ \\
\hline $\begin{array}{l}\text { Composites LPC }(70-80 \% \text { fibre de palmier dattier } / 20-30 \% \\
\text { matrice ; matrice }=\text { essence + Polystyrène, ratio massique } 1 \text { : } \\
\text { 3), Masri et al. }(2018) .\end{array}$ & $542-824$ & $0,12-0,76$ & $0,43-2,96$ \\
\hline $\begin{array}{l}\text { Composites Teck/polyester (composites moulés à froid, } 0 \text { - } \\
20 \% \text { colophane / } 5-20 \% \text { bois de Teck / polyester), Patel et } \\
\text { Rawat (2017). }\end{array}$ & $1045-1107$ & / & $5,90-7,34$ \\
\hline $\begin{array}{l}\text { PS-mix (composite extrudé, } 50 \% \text { mélange de déchets } \\
\text { plastiques traité avec solution saturée de polystyrène / } 50 \% \\
\text { fibres de feuilles de palmier dattier), Binhussain et El-Tonsy, } \\
\text { (2013). }\end{array}$ & 870 & 1,91 & / \\
\hline $\begin{array}{l}\text { MDF (medium density fiberboard), Binhussain et El-Tonsy } \\
\text { (2013). }\end{array}$ & $650-790$ & $1,35-2,5$ & $9,5-22$ \\
\hline
\end{tabular}


On observe sur la Figure 12 que le MOE et le MOR des CBPo changent lorsque changent les propriétés (module d'élasticité et contrainte à la rupture (Gérard et al., 2016)) de l'espèce de bois correspondant à la sciure utilisée. La bonne adhésion à l'interface boisPSE, ainsi mise en évidence, a été également observée par Masri et al. (2018) qui a utilisé une matrice similaire (à base de PSE et selon le même procédé que celui utilisé dans le présent travail) avec des fibres de branches de palmier dattier.

Par ailleurs, l'évaporation du solvant représente une source d'émission de composés organiques volatiles (COV). Dans une perspective de production industrielle, l'élimination de ces coV est indispensable non seulement pour la mise en œuvre de bonnes conditions d'hygiène et de sécurité mais également pour la préservation de l'environnement sous l'angle de la réduction des gaz à effet de serre. Dans ce sens, les auteurs du présent travail envisagent les solutions suivantes à mettre en œuvre sur la chaîne de fabrication des CBPo, celle-ci étant constituée principalement des étapes de préparation de la colle, préparation du composite, pré-séchage du composite, moulage et séchage des plaques. Pour l'hygiène et la sécurité des personnes, le port de masques appropriés dans les locaux de la chaîne de fabrication est indispensable. Aussi, en raison du caractère inflammable du solvant, toutes sources de flamme à l'intérieur ou dans le voisinage des locaux sont à proscrire. En ce qui concerne la préservation de l'environnement, la mise en place d'un système de collecte / élimination des COV est fortement recommandée. A ce titre, il peut être utilisé une hotte ou tout système $d^{\prime}$ aspiration des émissions aux différentes étapes de la chaîne de fabrication, associé à un système de ventilation des lieux de travail aménagés quasiment en vase clos. Un système assurant le recyclage, ou à défaut la combustion des COV captés doit être installé à la suite du système d'aspiration, pour permettre leur élimination.

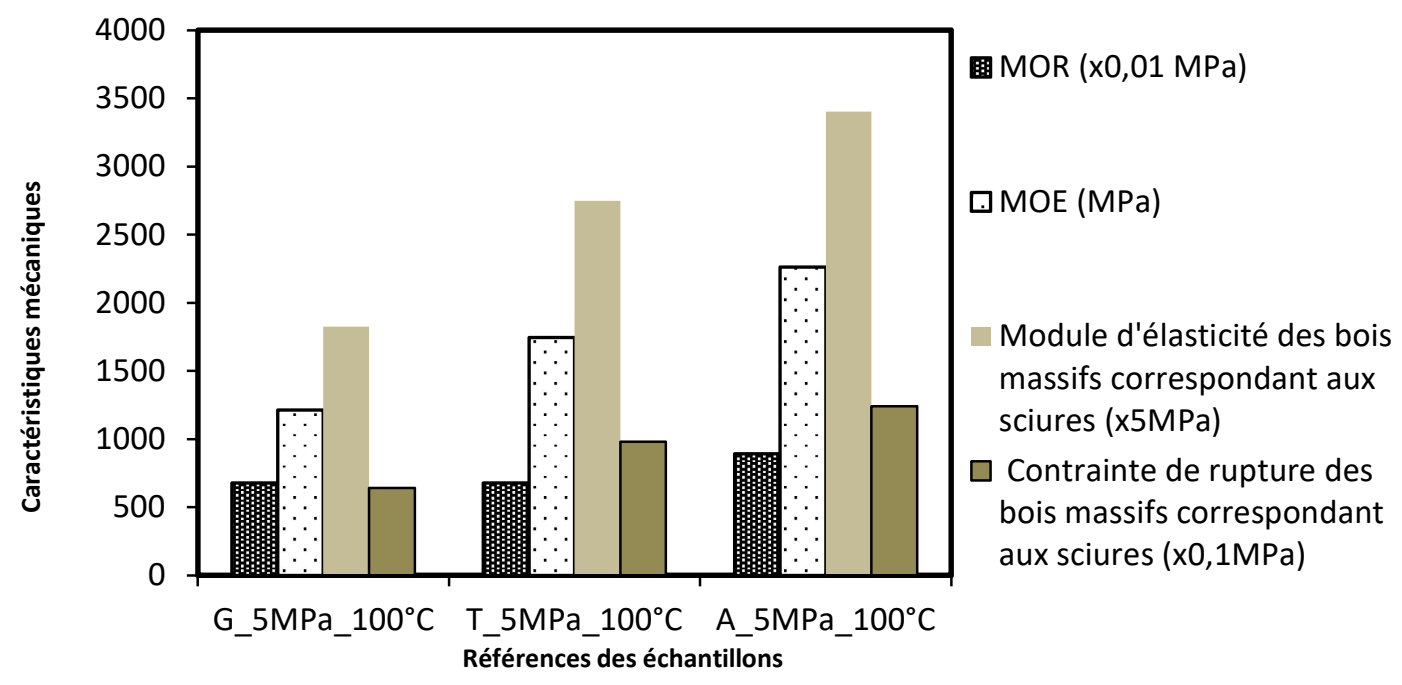

Figure 13. Valeurs comparées de MOE et MOR des CBPo à base de sciures de différentes espèces de bois, avec les modules et contraintes des bois massifs correspondants (G : Gmelina ; T : Teck ; A : Afzelia)

\subsection{Propriétés comparées de CBPO avec celles de matériaux similaires dans la littérature}

Les valeurs des propriétés des $\mathrm{CBPo}$ (à $25{ }^{\circ} \mathrm{C}$ et à $100^{\circ} \mathrm{C}$, pression de compactage $\geq 5 \mathrm{MPa}$ ) ainsi que celles de matériaux similaires sont présentées dans le tableau 3. La masse volumique des CBPo est de l'ordre de grandeur de la moyenne de chacun des différents matériaux rapportés de la littérature, excepté les composites bois de Teck/polyester de Patel et Rawat (2017) qui sont plus lourds. En particulier, elle est très proche de celle des MDF (Binhussain et El-Tonsy, 2013). Ceci montre que si les propriétés mécaniques des CBPo sont acceptables, on peut en envisager une multitude d'applications potentielle sur la base de leurs densités. La prise en compte de la pression et de la température a permis de repositionner les CBPo au niveau de certains matériaux présentés précédemment comme plus performants. Dans le tableau 3, on observe que les modules d'élasticité en flexion des CBPo formulés, $\mathrm{MOE}=0,6$ à 2,26 GPa, sont plus grands que ceux des composites LPC (Leaf Palm Composite) obtenus par Masri et al. (2018). Mais seul le CBPo A_5MPa_100 $\mathrm{C}$ a un module plus grand que celui de PS-mix de Binhussain et El-Tonsy, (2013). Les modules des CBPo sont similaires à ceux des MDF (Binhussain et ElTonsy, 2013). De plus, on observe dans le tableau 3 
que les contraintes maximums des CBPo formulés, $\mathrm{MOR}=2,71$ à $8,93 \mathrm{MPa}$, sont plus grandes que celles des composites LPC de Masri et al. (2018); en revanche, les contraintes maximums des échantillons G_7MPa_25 $5^{\circ} \mathrm{C} \quad$ A_5 $\mathrm{MPa}$ _ $100^{\circ} \mathrm{C}$, T_5MPa_ $100^{\circ} \mathrm{C}$, G_5MPa_ $100^{\circ} \mathrm{C}$ sont similaires à celles des composites de Patel et Rawat (2017). Le CBPo A_5MPa_ $100^{\circ} \mathrm{C}$ a une contrainte maximum proche de celle des MDF de MOR = 9,5 MPa (Binhussain et ElTonsy, 2013). Les différentes propriétés étudiées du CBPo A_5MPa_ $100^{\circ} \mathrm{C}$ sont similaires à celles des MDF de $M O R=9,5 \mathrm{MPa}$; une substitution entre les deux composites peut alors être envisagée.

\section{CONCLUSION}

L'analyse de la fabrication d'un composite à base de déchets d'industrie du bois et de polystyrène recyclé, élaboré suivant un procédé de moulage par compression à froid, a permis de mettre en évidence l'influence de deux paramètres de fabrication sur les propriétés mécaniques. Ainsi, la pression de compactage et la température sont des facteurs d'influence essentiels à considérer dans les plans d'expérience lors de l'évaluation des performances mécaniques des CBPo moulés par compression à froid. L'augmentation de la pression de compactage jusqu'à une valeur limite améliore significativement les propriétés mécaniques des CBPo. Les propriétés mécaniques optimales des CBPo ont été obtenues à la pression de 70 bars. Le séchage post formage à $100^{\circ} \mathrm{C}$, améliore également de façon significative les performances du composite. Du fait de leurs bonnes performances mécaniques, les CBPo se positionnent au même niveau que d'autres matériaux composites existants, permettant ainsi d'envisager de nouvelles applications dans la construction. Par ailleurs, dans une perspective de production industrielle, des études sont engagées en vue d'améliorer le procédé avec une meilleure prise en compte de la gestion des composés organiques volatiles émis lors de la fabrication du composite.

\section{REFERENCES BIBLIOGRAPHIQUES}

Agoua, E., Allognon-Houessou, E., Adjovi, E., Togbedji, B. (2013). Thermal conductivity of composites made of wastes of wood and expanded polystyrene. Construction and Building Materials 41, 557-562.

https://doi.org/10.1016/j.conbuildmat.2012.12.016

Almusawi, A.M., 2017. Mise en œuvre et optimisation des propriétés d'une structure sandwich en matériaux biosourcés (fibres et bois de chanvre) avec une matrice en polystyrène expansé pour le bâtiment. Matériaux. Thèse de Doctorat, Université Bourgogne Franche-Comté.
Animpong, M.A.B., Oduro, W.O., Koranteng, J., AmpomahBenefo, K., Boafo-Mensah, G., Akufo-Kumi, K., Tottimeh, G.O., Amoah, J.Y. (2017). Coupling effect of waste automotive engine oil in the preparation of wood reinforced LDPE plastic composites for panels. South African Journal of Chemical Engineering 24, 55-61. https://doi.org/10.1016/j.sajce.2017.01.004

Ansari, F., Granda, L.A., Joffe, R., Berglund, L.A., Vilaseca, F. (2017). Experimental evaluation of anisotropy in injection molded polypropylene/wood fiber biocomposites. Composites Part A: Applied Science and Manufacturing 96, 147-154.

https://doi.org/10.1016/j.compositesa.2017.02.003

Bessa, J., Mota, C., Cunha, F., Fangueiro, R. (2017). Influence of different thermoplastic polymer/wood ratios on the mechanical and thermal properties of composite materials. Procidia Engineering 200, 480-486.

Binhussain, M.A., El-Tonsy, M.M. (2013). Palm leave and plastic waste wood composite for out-door structures. Construction and Building Materials 47, 1431-1435. https://doi.org/10.1016/j.conbuildmat.2013.06.031

Borsoi, C., Berwig, K.H., Scienza, L.C., Zoppas, B.C.D.A., Brandalise, R.N., Zattera, A.J. (2014). Behavior in Simulated Soil of Recycled Expanded Polystyrene/Waste Cotton. Composites. Materials Research 17, 275-283. http://dx.doi.org/10.1590/S1516-14392013005000167

Borsoi, C., Scienza, L.C., Zattera, A.J. (2012).

Characterization of composites based on recycled expanded polystyrene reinforced with curaua fibers. Journal of Applied Polymer Science 128, 653-659. https://doi.org/10.1002/app.38236

Crevecoeur, G., Groeninckx, G. (1993). Sheet extrusion of in-situ composites_influence of processing parameters. Polymer Engineering \& Science 33, 937-943. https://doi.org/10.1002/pen.760331502

Gil-Jasso, N.D., Segura-González, M.A., Soriano-Giles, G., Neri-Hipolito, J., López, N., Mas-Hernández, E., BarreraDíaz, C.E., Varela-Guerrero, V., Ballesteros-Rivas, M.F. (2019). Dissolution and recovery of waste expanded polystyrene using alternative essential oils. Fuel 239, 611616. https://doi.org/10.1016/j.fuel.2018.11.055

Kokta, B.V., Chen, R., Daneault, C., Valade, J.L. (1983). Use of wood fibers in thermoplastic composites. Polymer Composites 4, 229-232.

https://doi.org/10.1002/pc.750040407

Le Duigou, A., Castro, M., Bevan, R., Martn, N. (2016). 3D printing of wood fibre biocomposites From mechanical to actuation functionality. Materials \& Design 96, 106-114. https://doi.org/10.1016/j.matdes.2016.02.018

Madsen, B., 2004. Properties of plant fiber yarn polymer composites: an experimental study (Ph. D. Thesis). Technical University of Denmark, Kgs. Lyngby.

Mangalara, S.C.H., Varughese, S. (2016). A green recycling approach to obtain nano and microparticles from expanded polystyrene waste. ACS Sustainable Chemistry \& Engineering 4, 6095-6100.

https://doi.org/10.1021/acssuschemeng.6b01493 
Masri, T., Ounis, H., Sedira, L., Kaci, A., Benchabane, A.

(2018). Characterization of new composite material based on date palm leaflets and expanded polystyrene wastes. Construction and Building Materials 164, 410-418. https://doi.org/10.1016/j.conbuildmat.2017.12.197

Matuana, L.M., Stark, N.M. (2015). The use of wood fi bers as reinforcements in composites. Biofiber reinforcements in composites materials, 648-688.

https://doi.org/10.1533/9781782421276.5.648

O’Dowd, F., Lévesque, M., Gilchrist, M.D. (2006). Analysis of fibre orientation effects on injection moulded components. Proceedings of the Institution of Mechanical Engineers, Part B: Journal of Engineering Manufacture 220, 1909-1921. https://doi.org/10.1243/09544054JEM332

Patel, V.K., Rawat, N., (2017). Physico-mechanical properties of sustainable Sagwan-Teak Wood Flour/Polyester Composites with/without gum rosin. Sustainable Materials and Technologies 13, 1-8. https://doi.org/10.1016/j.susmat.2017.05.002

Poletto, M., Dettenborn, J., Zeni, M., Zattera, A.J. (2011). Characterization of composites based on expanded polystyrene wastes and wood flour. Waste Management, 31, 779-784.

https://doi.org/10.1016/j.wasman.2010.10.027

Sallih, N., Lescher, P., Bhattacharyya, D. (2014). Factorial study of material and process parameters on the mechanical properties of extruded kenaf fibre_polypropylene composite sheets. Composite Part A: Applied Science and Manufacturing 61, 91-107. https://doi.org/10.1016/j.compositesa.20140.02.014

Stark, N.M., Rowlands, R.E. (2003). Effects of wood fiber characteristics on mechanical properties of wood/polypropylene composites. Wood Fiber Science, 35, 167-174.

Wong, S., Lee, W.S., Naguib, H.E., Park, C.B. (2008). Effect of processing parameters on the mechanical properties of injection molded thermoplastic polyolefin (TPO) cellular foams. Macromolecular Material and Engineering, 293, 605-613. 